\title{
Diagnostic yield of quantitative endotracheal aspirates in patients with severe nursing home-acquired pneumonia
}

\author{
Ali A El Solh, Morohunfolu E Akinnusi, Lilibeth A Pineda and Corey R Mankowski
}

\author{
Western New York Respiratory Research Center, Division of Pulmonary, Critical Care, and Sleep Medicine, Department of Medicine, State University \\ of New York at Buffalo School of Medicine and Biomedical Sciences, Grider Street Buffalo, New York 14215, USA \\ Corresponding author: Ali A El Solh, solh@buffalo.edu
}

Received: 26 Mar 2007 Accepted: 17 May 2007 Published: 17 May 2007

Critical Care 2007, 11:R57 (doi:10.1186/cc5917)

This article is online at: http://ccforum.com/content/11/3/R57

(c) 2007 El Solh et al.; licensee BioMed Central Ltd.

This is an open access article distributed under the terms of the Creative Commons Attribution License (http://creativecommons.org/licenses/by/2.0), which permits unrestricted use, distribution, and reproduction in any medium, provided the original work is properly cited.

\begin{abstract}
Introduction Diagnostic strategies based on tracheal aspirates in patients with severe nursing home-acquired pneumonia have not previously been evaluated. The objectives of the study were to investigate, in patients with severe nursing home-acquired pneumonia, the diagnostic value of quantitative endotracheal aspirate (QEA) cultures using increasing interpretative cutoff points, as compared with bronchoalveolar lavage (BAL) and protected specimen brush (PSB) quantitative cultures.
\end{abstract}

Methods Seventy-five nursing home patients requiring mechanical ventilation for suspected pneumonia were studied. Endotracheal aspirate, PSB, and BAL samples were obtained consecutively. The diagnostic yield of QEA at thresholds raging from $10^{3}$ to $10^{7}$ colony-forming units (cfu)/ml was assessed by calculating sensitivities, specificities, and accuracy rates. A receiver operator characteristic curve for the series of cutoff points was constructed.
Results Forty-nine patients were diagnosed with pneumonia either by BAL $\left(\leq 10^{4} \mathrm{cfu} / \mathrm{ml}\right)$ or PSB $\left(\leq 10^{3} \mathrm{cfu} / \mathrm{ml}\right)$. Diagnostic accuracy of QEA was most favorable at $10^{4} \mathrm{cfu} / \mathrm{ml}$. At this threshold, endotracheal aspirates coincided with both BAL and PSB in 30 cases, whereas partial agreement was observed in 14 cases. This resulted in sensitivity and specificity of $90 \%$ (95\% confidence interval $78 \%$ to $97 \%$ ) and $77 \%$ (95\% confidence interval $56 \%$ to $91 \%$ ), respectively. OEA findings correlated significantly with both $\mathrm{PSB}$ and BAL quantitative cultures $(r=0.71[P<0.001]$ and $r=0.77[P<0.001]$, respectively).

Conclusion QEA may be used as a diagnostic tool to determine the presence of pneumonia in ventilated patients admitted from nursing homes when bronchoscopic procedures are not feasible or available.

\section{Introduction}

Nursing home-acquired pneumonia (NHAP) is the leading cause of death among long-term care patients and is one of the most common causes of transfer to hospital, where it accounts for $2 \%$ to $18 \%$ of patients hospitalized for pneumonia $[1,2]$. The overall mortality rate among patients requiring hospitalization for NHAP ranges from $13 \%$ to $41 \%[3,4]$ and rises to $57 \%$ in those with severe NHAP requiring treatment in the intensive care unit [5].

Evaluation of the epidemiology of NHAP has been limited by low bacteriologic yield, poor quality of respiratory specimens, and potential contamination of the upper respiratory tract $[6,7]$. Many nursing home residents have extensive co-morbid- ities, which increase their risk for colonization with Staphylococcus aureus or enteric Gram-negative rods. However, there is no consensus regarding the best diagnostic strategy in these patients. Clinical features of NHAP are unreliable in establishing accurately the diagnosis of pneumonia in such patients. Quantitative cultures of protected specimen brush (PSB) and bronchoalveolar lavage (BAL) specimens have been used to diagnose severe NHAP $[5,8]$. However, use of these techniques has been limited by the fact that they are invasive, time consuming, and require fiberoptic bronchoscopy to be available 24 hours per day. Use of quantitative endotracheal aspirates (QEAs) in intubated patients could be viewed as a simple, fast, and cheap way to identify the responsible microorganisms and, combined with knowledge of local 
epidemiologic characteristics, can promote early use of appropriate antibiotic therapy. However, the accuracy of QEA in this particular setting has never been assessed. All comparisons of QEAs with other techniques were conducted in patients with ventilator-associated pneumonia [9-11]. The aim of the present study was to compare the diagnostic accuracy of post-intubation QEA with those of PSB and BAL in patients requiring mechanical ventilation for suspected NHAP.

\section{Materials and methods Study population}

The study was conducted in a University-affiliated tertiary care center and was derived from a prospective database developed over a six year period extending from January 2000 to December 2005. All nursing home residents presenting to the emergency room with a diagnosis of pneumonia requiring mechanical ventilation were eligible for enrollment if a radiographic infiltrate was present that was compatible with pneumonia, together with symptoms suggestive of lower respiratory tract infection, and any two of the following clinical parameters were present: temperature $\leq 38^{\circ} \mathrm{C}$ or $<36^{\circ} \mathrm{C}$; white blood cells $\leq 10,000 / \mathrm{mm}^{3}$ or $<4,000 / \mathrm{mm}^{3}$, or $>15 \%$ immature neutrophils (bands) regardless of total peripheral white blood cell count; and purulent respiratory secretions. Patients with severe immunosuppression (solid organ transplantation or steroid therapy $\leq 20 \mathrm{mg} /$ day for more than two weeks), witnessed aspiration, or in whom the pneumonia was an expected terminal event resulting from metastatic cancer were excluded. In addition, patients who were receiving antimicrobial therapy within 72 hours before enrollment were also excluded. The study was approved by the institutional review board and, informed consent was obtained from all participants' next of kin before any invasive procedure was performed.

\section{Data collection}

Data collected on intensive care unit admission included age, sex, co-morbid illnesses, Charlson Index [12], clinical symptoms, and chest radiographic pattern and distribution. The most deranged readings of vital signs, laboratory data, and blood gas analysis within the first 24 hours of admission were also recorded.

\section{Specimen collection}

Within two hours of enrollment in the study, all patients underwent the following protocol. Intravenous midazolam was given before the procedures. The fraction of inspired oxygen was set at $90 \%$ or more. None of the patients received local anesthetics. A blind endotracheal aspiration sample was obtained first by sterile means using a 22-inch suction catheter and collected in a mucus collector (Specimen trap, Busse, Hauppauge, NY, USA). Subsequently, a bronchoscopic PSB procedure was performed. A PSB sample was retrieved from the area of maximal inflammation and purulence, as suspected based on chest roentgenogram findings. BAL was then per- formed from the same segment where PSB had been conducted and analyzed according to standard criteria. All retrieved specimens (endotracheal aspirate, BAL fluid, and PSB specimen) were sent to the microbiology laboratory immediately after collection.

\section{Microbiologic processing}

Endotracheal aspirate and BAL samples were mechanically liquefied and homogenized by vortexing for $1 \mathrm{~min}$ with glass beads, followed by centrifuging at 3,000 rpm for $10 \mathrm{~min}$. PSB samples were aseptically cut and placed in a sterile tube containing $1 \mathrm{ml}$ of $0.9 \%$ saline solution and vortexed for $1 \mathrm{~min}$. All three types of specimens were serially diluted in $0.9 \%$ sterile saline solution. The specimens were then plated into the following agar media: 5\% sheep blood, chocolate, and McConkey agar. All cultures were incubated at $37^{\circ} \mathrm{C}$ under aerobic and anaerobic conditions and in a carbon dioxide enriched atmosphere. Plates were evaluated for growth at 24 and 48 hours and discarded after five days. The number of bacteria in the original sample was expressed in colony-forming units (cfu) per milliliter. All micro-organisms were identified using standard laboratory methods. The threshold for positive culture defining pneumonia was $10^{3} \mathrm{cfu} / \mathrm{ml}$ for PSB or $10^{4} \mathrm{cfu} / \mathrm{ml}$ for BAL, or both. The cutoff points analyzed to establish a positive result for QEA were $10^{3}, 10^{4}, 10^{5}, 10^{6}$, and $10^{7} \mathrm{cfu} / \mathrm{ml}$.

\section{Statistical analysis}

Descriptive analysis was performed using the NCSS 2000 software (NCSS Statistical Analysis System, Kaysville, UT, USA). Means were compared using the Student's $t$-test when they were normally distributed and the Mann-Whitney test otherwise. Frequencies were compared using a $\chi^{2}$ test or Fisher's exact test when appropriate. Sensitivity, specificity, false-positive rate, false-negative rate, and overall accuracy were calculated using the standard formulae [13]. Quantitative concordance between techniques was assessed with Pearson's correlation coefficient. Graphic representation of diagnostic parameters was performed, plotting true-positive against false-positive percentages to obtain a receiver operator characteristic curve. All tests were two tailed, and statistical significance was determined at the $5 \%$ level.

\section{Results}

Seventy-five patients (45 men and 30 women) were included in the study. The general characteristics of the study population are shown in Table 1. All patients were mechanically ventilated at the time of enrollment. Twenty-one patients had received at least one dose of antimicrobial agent before bronchial samplings.

According to our diagnostic criteria, pneumonia was documented in 49 (65\%) patients. Fifty-six micro-organisms were isolated at concentrations above the diagnostic thresholds by either PSB or BAL (Table 2). Polymicrobial infection was present in three patients. The most frequently isolated bacteria 
Table 1

\begin{tabular}{ll}
\hline Clinical characteristics of the study population \\
\hline Characteristic & Value \\
\hline Age (years) & $72.7 \pm 14.9$ \\
Sex (male/female) & $45 / 30$ \\
Comorbid diseases & \\
Coronary artery disease & $15(20)$ \\
Chronic pulmonary & $31(41)$ \\
obstructive disease & $11(15)$ \\
Diabetes mellitus & $27(36)$ \\
Cerebrovascular accident & $2.3 \pm 1.4$ \\
Charlson Index & \\
Clinical presentation & $59(79)$ \\
Fever & $73(97)$ \\
Dyspnea & $32(43)$ \\
Cough & $27.8 \pm 5.7$ \\
$\mathrm{APACHE}_{\text {II score }}$ & $241 \pm 56$ \\
$\mathrm{PaO}$ & /FiO \\
$\mathrm{Radiologic}_{2}$ & $4.6 \pm 1.8$ \\
\hline
\end{tabular}

Seventy-five patients were included. Values are expressed as number, number (\%), or mean \pm standard error. APACHE, Acute Physiology and Chronic Health Evaluation; $\mathrm{FiO}_{2}$, fractional inspired oxygen; $\mathrm{PaO}_{2}$, arterial oxygen tension;

were Staphylococcus aureus $(n=13)$, Streptococcus pneumoniae $(n=7)$, and Escherichia coli $(n=7)$. There was total agreement in positive bacterial cultures between PSB and BAL in 33 cases. Twenty-six paired cultures were sterile, and in 19 of these cases there was exposure to antimicrobial therapy before the invasive procedures were conducted. Discrepancy was noted in 16 cases, which represented 11 BALpositive/PSB-negative cases and five BAL-negative/PSB-positive cases.

The operating characteristics of QEA for detecting the presence of pneumonia were obtained over a range of values extending from $10^{3}$ through to $10^{7} \mathrm{cfu} / \mathrm{ml}$ (Table 3 and Figure
Table 2

Results of quantitative bacterial cultures of BAL and PSB

\begin{tabular}{lcc}
\hline Pathogen & BAL $\left(\leq 10^{4}\right)$ & PSB $\left(\leq 10^{3}\right)$ \\
\hline $\begin{array}{l}\text { Staphylococcus } \\
\text { aureus }\end{array}$ & 12 & 8 \\
$\begin{array}{l}\text { Streptococcus } \\
\text { pneumoniae }\end{array}$ & 6 & 4 \\
$\begin{array}{l}\text { Streptococcus spp. } \\
\begin{array}{l}\text { Haemophilus } \\
\text { influenzae }\end{array}\end{array}$ & 5 & 4 \\
Klebsiella spp. & 3 & 2 \\
$\begin{array}{l}\text { Escherichia coli } \\
\text { Serratia marcescens }\end{array}$ & 5 & 5 \\
Proteus mirabilis & 6 & 6 \\
Enterobacter ssp. & 3 & 3 \\
$\begin{array}{l}\text { Pseudomonas } \\
\text { aeruginosa }\end{array}$ & 4 & 2 \\
\hline
\end{tabular}

BAL, bronchoalveolar lavage; PSB, protected specimen brush.

1). The sensitivity increased from $18 \%$ at the threshold of $10^{7}$ $\mathrm{cfu} / \mathrm{ml}$ to $98 \%$ at $10^{3} \mathrm{cfu} / \mathrm{ml}$. Conversely, specificity decreased from $100 \%$ at $10^{7} \mathrm{cfu} / \mathrm{ml}$ to $35 \%$ at $10^{3} \mathrm{cfu} / \mathrm{ml}$. The threshold of $10^{4} \mathrm{cfu} / \mathrm{ml}$ yielded the highest accuracy in diagnosis, with a sensitivity of $90 \%$ (95\% confidence interval [Cl] 78\% to $97 \%$ ) and a specificity of $77 \%$ ( $95 \% \mathrm{Cl} 56 \%$ to $91 \%$ ). At this threshold, the positive-predictive value was $88 \%(95 \% \mathrm{Cl} 76 \%$ to $96 \%)$ and the negative predictive value was $80 \%(95 \% \mathrm{Cl}$ $59 \%$ to $93 \%)$.

Using $10^{4} \mathrm{cfu} / \mathrm{ml}$ as a diagnostic threshold for endotracheal aspirates, QEA coincided with both BAL and PSB in 30 positive culture cases. Partial agreement was present in 14 cases; 10 patients had qualitative concordance between QEA and $B A L$ and four others between QEA and PSB only. In six cases QEA was below the threshold of $10^{4} \mathrm{cfu} / \mathrm{ml}$ for pneumonia documented by BAL or PSB. Comparisons of bacterial counts between endotracheal aspirate and BAL and between endotracheal aspirate and PSB are shown in Figures 2 and 3.

Table 3

Operating characteristics of the endotracheal aspirates quantitative cultures

\begin{tabular}{llll}
\hline Threshold $(\mathrm{cfu} / \mathrm{ml})$ & Sensitivity (\%) & Specificity (\%) & Accuracy (\%) \\
\hline$\leq 10^{3}$ & $98(89-100)$ & $35(17-56)$ & $76(65-85)$ \\
$\leq 10^{4}$ & $90(78-97)$ & $77(56-91)$ & $85(78-94)$ \\
$\leq 10^{5}$ & $78(63-88)$ & $84(65-96)$ & $80(69-88)$ \\
$\leq 10^{6}$ & $51(36-66)$ & $92(75-99)$ & $65(54-76)$ \\
$\leq 10^{7}$ & $18(9-32)$ & $100(87-100)$ & $47(35-59)$ \\
\hline
\end{tabular}

Values in parentheses are 95\% confidence intervals. 
Figure 1

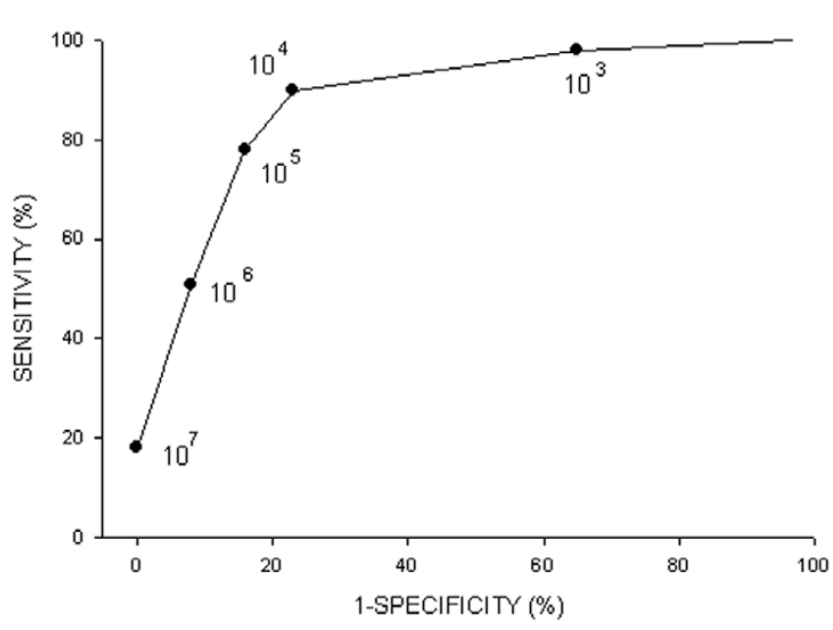

Receiver operator characteristic curve of QEA for the diagnosis of pneumonia. OEA, quantitative endotracheal aspirate.

The correlation coefficients $(r)$ between the quantitative cultures of micro-organisms obtained from endotracheal aspirate and BAL and from endotracheal aspirate and PSB were 0.77 $(P<0.001)$ and $0.71(P<0.001)$, respectively.

\section{Discussion}

The results of this study indicate that the best overall accuracy in diagnosing severe NHAP using the endotracheal aspirate culture technique was obtained at a threshold of $10^{4} \mathrm{cfu} / \mathrm{ml}$. At this cutoff, there was favorable correlation between endotracheal aspirate and PSB cultures and between endotracheal aspirate and BAL cultures. These findings suggest that $\mathrm{QEA}$ can be used as a diagnostic tool in severe NHAP where fiberoptic bronchoscopic techniques are not uniformly available or are not practically feasible before the first dose of antimicrobial therapy.

NHAP has traditionally been approached as a disease that resembles community-acquired pneumonia rather than nosocomial pneumonia. The lack of strong epidemiologic data on microbial etiology has resulted in physicians employing diagnostic and treatment strategies in NHAP that are similar to those used in community-acquired pneumonia, and with similar empiric antibiotic coverage. Following the publication of several investigations on the microbial spectrum of pathogens in NHAP [5,14], this approach has shifted drastically toward classifying NHAP as health care-associated pneumonia. Because of the substantial mortality associated with both inadequate and delayed therapy $[5,15,16]$, the most recent American Thoracic Society guideline [17] advises that the initial empirical antibiotic coverage for patients hospitalized with NHAP should have adequate activity against multidrug resistant pathogens. However, indiscriminate use of broadspectrum antibiotics has been implicated in the emergence of resistant strains in the intensive care unit $[18,19]$. In response
Figure 2

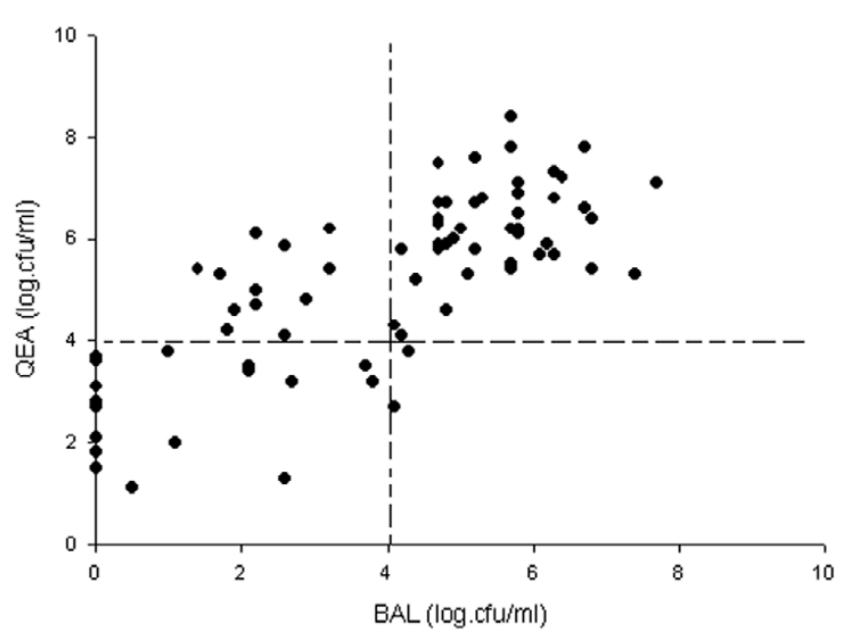

Correlation between quantitative cultures of endotracheal aspirate and BAL specimens. The correlation coefficient $(r)$ is $0.77(P<0.001)$. $\mathrm{BAL}$, bronchoalveolar lavage; cfu, colony-forming units; $\mathrm{QEA}$, quantitative endotracheal aspirate.

to this, a consensus has emerged emphasizing the implementation of de-escalation therapy or discontinuation of antibiotics once culture results and susceptibility findings are available. Hitherto, the concept of de-escalation has not been tested in patients with severe NHAP, in part because of lack of studies to determine appropriate diagnostic strategies in this segment of the population.

This is the first study to our knowledge to assess the accuracy of QEA, as compared with those of PSB and BAL, in the diagnosis of severe NHAP. Previous studies established that cultures of tracheal aspirates in patients requiring mechanical ventilation for longer than 48 hours may not be accurate [20].

Figure 3

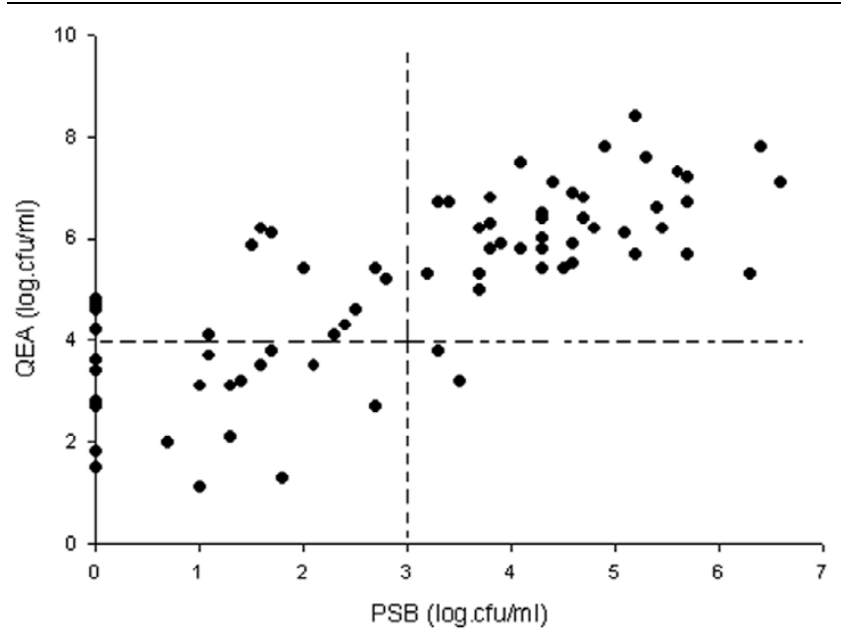

Correlation between quantitative cultures of endotracheal aspirate and PSB specimens. The correlation coefficient $(r)$ is $0.71(P<0.001)$. PSB, protected specimen brush; cfu, colony-forming units; $Q E A$, quantitative endotracheal aspirate. 
Although negative cultures may have high predictive value, the rate of false-positive results can be high, leading to over-diagnosis of pneumonia and misdiagnosis of etiology. Similarly, quantitative cultures of tracheal aspirates have also yielded mixed results. In intubated patients suspected with nosocomial pneumonia, a colony count $\leq 10^{4} \mathrm{cfu} / \mathrm{ml}$ was found in $80 \%$ of patients with clinical pneumonia but also in $61 \%$ of patients without obvious pneumonia [11]. These findings stem from the fact that endotracheal tube colonization and biofilm formation occurs in many patients undergoing mechanical ventilation from an early stage. The absence of such a risk factor would minimize the risk for tracheal aspirate contamination, resulting in improved diagnostic accuracy. Indeed, at a threshold of $10^{4} \mathrm{cfu} / \mathrm{ml}$, we were able to demonstrate good sensitivity $(90 \%)$, specificity $(77 \%)$, and negative predictive value (80\%) for QEA as compared with BAL and PSB. It is noteworthy that one-third of our patients had received antibiotics before samplings, which could be regarded as a potential bias because it could have led to a high rate of false-negative results. Lowering the threshold value has been proposed as an alternative for patients receiving antibiotic therapy in patients suspected of having ventilator-associated pneumonia [21]. In this study we were unable to confirm this approach, in part because of the lack of histopathologic specimens.

One of the major advantages of endotracheal cultures is that they exclude certain types of infection when the organism is absent [22]. For example, absence of Pseudomonas in an endotracheal aspirate makes it unlikely that this organism is the cause of an infection. Conversely, it could be argued that the lack of micro-organisms on Gram staining in nursing home patients suspected of having NHAP might suggest the presence of atypical pathogens. Although outbreaks of pneumonia caused by atypical pathogens can occur among nursing home residents, the incidence of these infections is relative low in this population $[5,23]$. Nonetheless, appropriate testing should be performed and empiric coverage should be provided when it is highly suspected.

This study has a number of strengths, including use of each patient as his or her own control and using explicitly defined criteria for quantitative culture positivity. However, it has a number of limitations, as do all studies in this discipline. We relied upon bronchoscopic techniques (PSB and BAL) as gold standards, but these methods are not $100 \%$ sensitive or specific. It has been suggested that only the combined results of histologic examination and quantitative cultures of lung tissue are sufficiently powerful to rule in or rule out the presence of pneumonia. Clearly, such a highly invasive sampling approach would not be practical in a frail population. Without an indisputable and easily obtainable reference, calculations of sensitivity and specificity will remain problematic. Finally, the impact of QEA findings on antibiotic therapy and patient outcomes was not assessed. Pending further validation, we thought it would be too premature to make any antibiotic rec- ommendation for the management of severe NHAP based on the present study alone.

\section{Conclusion}

The present study shows that QEA using a cutoff point of $10^{4}$ $\mathrm{cfu} / \mathrm{ml}$ represents a practical diagnostic method in patients with severe NHAP. This modality may be useful as a first line technique for intubated NHAP patient with clinical suspicion of pneumonia when it is more convenient to perform prior to administration of antibiotic therapy.

\section{Key messages}

- Accurate diagnosis of severe NHAP remains a challenge for clinicians in intensive care unit settings.

- Quantitative cultures of samples obtained by BAL and PSB are considered to be the tests that offer the best diagnostic accuracy, but these methods are invasive, expensive, and not uniformly available.

- Quantitative endotracheal aspirates in cases of severe NHAP correlate well with quantitative bronchoscopic $P S B$ and BAL in intubated nursing home patients who are suspected of having clinical pneumonia.

- QEA may be a reliable alternative to $\mathrm{PSB}$ or $\mathrm{BAL}$ in the setting of severe NHAP.

\section{Competing interests}

The authors declare that they have no competing interests.

\section{Authors' contributions}

AES conceived of the study, collected all respiratory samples, and edited the manuscript. MEA participated in data collection and data analysis. LAP assisted with the acquisition of the respiratory samples, performed quality assurance, and provided a first draft of the manuscript. CRM recruited eligible patients, and collected clinical and microbiologic data.

\section{References}

1. Gross JS, Neufeld RR, Libow LS, Gerber I, Rodstein M: Autopsy study of the elderly institutionalized patient. Review of $\mathbf{2 3 4}$ autopsies. Arch Intern Med 1988, 148:173-176.

2. Marrie T, Durant H, Yates L: Community-acquired pneumonia requiring hospitalization: 5-year prospective study. Rev Infect Dis 1989, 11:586-599.

3. Loeb M, McGeer A, McArthur M, Walter S, Simor AE: Risk factors for pneumonia and other lower respiratory tract infections in elderly residents of long-term care facilities. Arch Intern Med 1999, 159:2058-2064.

4. Mehr DR, Zweig SC, Kruse RL, Popejoy L, Horman D, Willis D, Doyle ME: Mortality from lower respiratory infection in nursing home residents: a pilot prospective community-based study. $J$ Fam Pract 1998, 47:298-304.

5. El Solh A, Sikka P, Ramadan F, Davies J: Etiology of severe pneumonia in the very elderly. Am J Respir Crit Care Med 2001, 163:645-651.

6. Barrett-Connor $\mathrm{E}$ : The nonvalue of sputum culture in the diagnosis of pneumococcal pneumonia. Am Rev Respir Dis 1971, 103:845-848. 
7. Davidson M, Tempest B, Palmer DL: Bacteriologic diagnosis of acute pneumonia: comparison of sputum, transtracheal aspirates, and lung aspirates. JAMA 1976, 235:158-163.

8. El Solh A, Aquilina A, Dhillon R, Ramadan F, Nowak P, Davies J: Antimicrobial treatment failure in institutionalized elderly with severe pneumonia. Am J Respir Crit Care Med 2002, 166:1038-1043.

9. Marquette $\mathrm{C}$, Georges $\mathrm{H}$, Wallet $F$, Ramon $\mathrm{P}$, Saulnier F, Neviere $R$, Mathieu $D$, Rime A, Tonnel A: Diagnostic efficiency of endotracheal aspirates with quantitative bacterial cultures in intubated patients with suspected pneumonia. Am Rev Respir Dis 1993, 148:138-144.

10. El-Ebiary M, Torres A, Gonzalez J, Puig De La Bellacasa J, Garcia C, Jimenez de Anta M, Ferrer M, Rodriguez-Roisin R: Quantitative cultures of endotracheal aspirates for the diagnosis of ventilator-associated pneumonia. Am Rev Respir Dis 1993, 148:1552-1557.

11. Jourdain B, Novara A, Joly-Guillou M, Dombret M, Calvat S, Trouillet J, Gibert C, Chastre J: Role of quantitative cultures of endotracheal aspirates in the diagnosis of nosocomial pneumonia. Am J Respir Crit Care Med 1995, 152:241-246.

12. Charlson ME, Pompei $P$, Ales KL, MacKenzie CR: A new method of classifying prognostic co-morbidity in longitudinal studies: development and validation. J Chron Dis 1987, 40:373-383.

13. Griner P, Mayewski R, Mushlin A, Greenland P: Selection and interpretation of diagnostic tests and procedures. Principles and applications. Ann Intern Med 1981, 94:557-592.

14. Kollef M, Shorr A, Tabak Y, Gupta V, Liu L, Johannes R: Epidemiology and outcomes of health care associated pneumonia. Chest 2005, 128:3854-3862.

15. Fagon JY, Chastre J: Management of suspected ventilatorassociated pneumonia. Ann Intern Med 2000, 133:1009.

16. Houck PM, Bratzler DW, Nsa W, Ma A, Bartlett J: Timing of antibiotic administration and outcomes for Medicare patients hospitalized with community-acquired pneumonia. Arch Intern Med 2004, 164:637-644.

17. American Thoracic Society/Infectious Diseases Society of America: Guidelines for the management of adults with hospitalacquired, ventilator-associated, and health care-associated pneumonia. Am J Respir Crit Care Med 2005, 171:388-416.

18. Corbella $X$, Montero A, Pujol M, Dominguez MA, Ayats J, Argerich MJ, Garrigosa F, Ariza J, Gudiol F: Emergence and rapid spread of carbapenem resistance during a large and sustained hospital outbreak of multiresistant Acinetobacter baumannii. J Clin Microbiol 2000, 38:4086-4095.

19. Trouillet JL, Vuagnat A, Combes A, Kassis N, Chastre J, Gibert C: Pseudomonas aeruginosa ventilator-associated pneumonia: comparison of episodes due to piperacillin-resistant vs. piperacillin-susceptible organisms. Clin Infect Dis 2002, 34:1047-1054

20. Johanson W, Seidenfeld J, Gomez P, Los Santos R, Coalson J: Bacteriologic diagnosis of nosocomial pneumonia following prolonged mechanical ventilation. Am Rev Respir Dis 1988, 137:259-264.

21. Timsit JF, Misset B, Renaud B, Goldstein FW, Carlet J: Effect of previous antimicrobial therapy on the accuracy of the main procedures used to diagnose nosocomial pneumonia in patients who are using ventilation. Chest 108:1036-1040.

22. San Pedro G: Are quantitative cultures useful in the diagnosis hospital-acquired pneumonia? Chest 2001, 119:385S-390S.

23. Orr PH, Peeling RW, Fast M, Brunka J, Duckworth H, Harding GK, Nicolle LE: Serological study of responses to selected pathogens causing respiratory tract infection in the institutionalized elderly. Clin Infect Dis 1996, 23:1240-1245. 